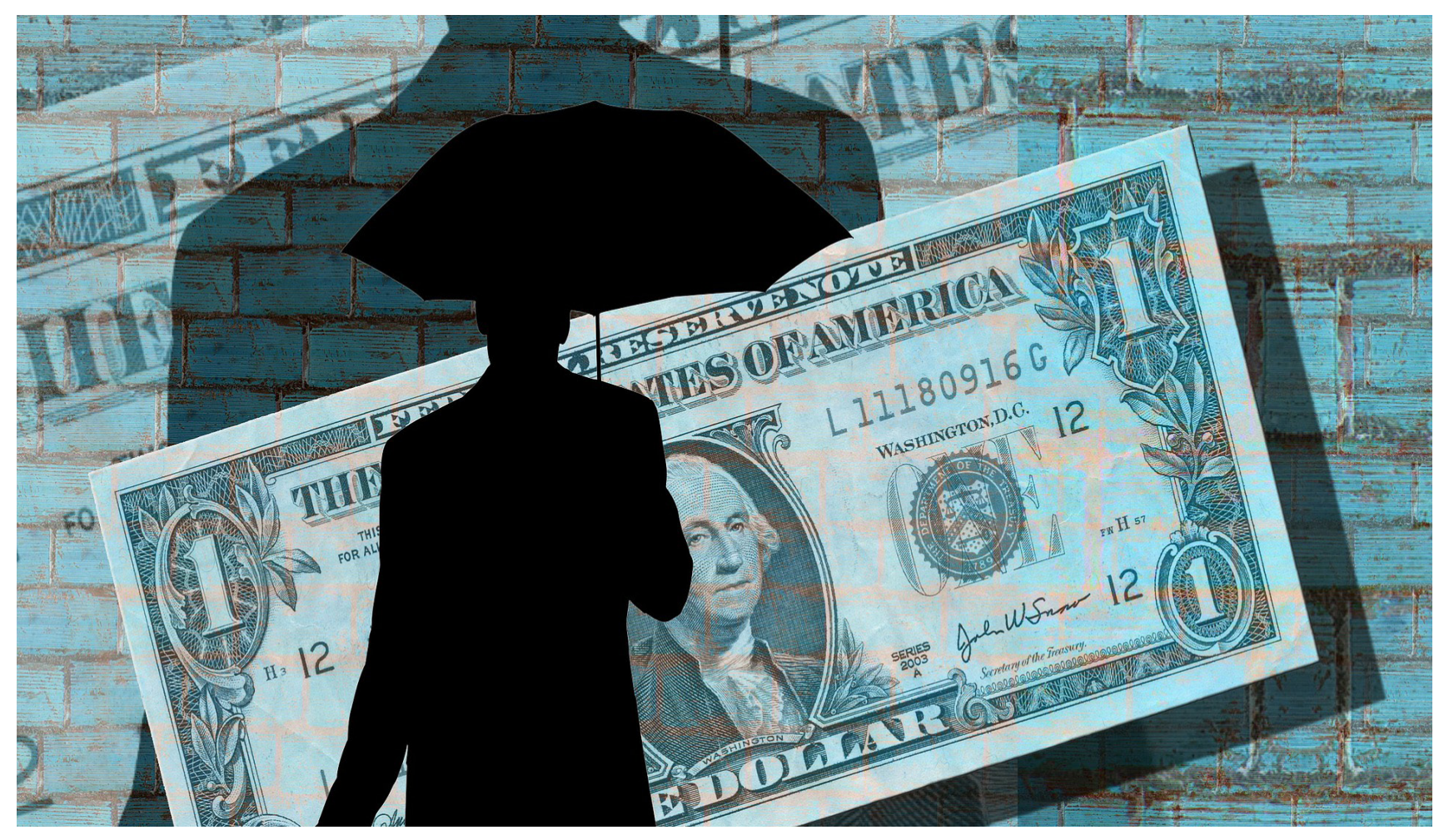

\title{
Análisis de las reformas tributarias en Colombia de los años 2018 a 2020
}

\section{Analysis of the tax reforms in Colombia from 2018 to 2020}

Evelyn Julieth Pacheco-Moncada

Estudiantes del Programa de Contaduría Pública, Universidad Francisco de Paula Santander, Cúcuta, Colombia

\section{Diego Fernando Leal-Correa}

Estudiantes del Programa de Contaduría Pública, Universidad

Francisco de Paula Santander, Cúcuta, Colombia
Carlos Fabián Rico-Rojas

Candidato a Doctor en Gerencia Pública y Política Fiscal, carlosfabianrr@ufps.edu.co, Universidad Francisco de Paula Santander, Cúcuta, Colombia 


\section{Resumen}

El objetivo de esta investigación es analizar los cambios que traen consigo las reformas tributarias en el país, las problemáticas que estas generan y por las cuales se crean nuevas normas tributarias. Se realizó una recopilación de historia de ella, causas que impulsan la alteración de estas e impactos que han tenido en nuestro país en estos últimos años, por ello tuvimos en cuenta repositorios de universidades, revistas, diferentes sitios web y artículos. En Colombia cada año se realiza cambios en las normas tributarias, ya que en muy pocas ocasiones se llega a recoger el dinero que tienen planeado y esto sucede, por la falta de información de las personas tanto naturales como jurídicas, puesto que no pagan los impuestos correspondientes, se las ingenian para una evasión de estos, sin embargo, no sólo son los ciudadanos, porque la mayoría de estos si cumplen con su deber, también es el estado que en gran parte es muy corrupto y no tiene una planeación a largo plazo con los nuevos impuesto que agrega o retira, por consiguiente, hay cambios en estas normas, dado que no cumplen con su fin que es estimular el crecimiento del país, la equidad y dar un apoyo a todos los empresarios para el fomento del empleo. Para concluir, evidenciamos que con el último gobierno de Iván Duque se ha cumplido con algunos de los cambios propuestos en las reformas, en cambio, ha hecho reforma por año cosa que no beneficia al país, porque no se adaptan a la primera reforma, cuando ya está ingresando la segunda. Sin embargo, este gobierno ha pasado por la crisis económica que ha traído consigo el Covid-19 y tiene en planes su tercera reforma, visto que el dinero recogido fue invertido para el bienestar de los ciudadanos y su sustento mientras sucedía la pandemia. Se necesita para la nación una reforma tributaria estructural, pero que esta tenga visión a largo plazo.

Palabras clave: Reformas tributarias, efectos, ley de financiamiento, Colombia.

\section{Abstract}

The objective of this research is to analyze the changes brought about by tax reforms in the country, the problems they generate and for which new tax regulations are created. A compilation of its history was made, causes that promote the alteration of these and impacts that they have had in our country in recent years, for this reason we took into account repositories of universities, magazines, different websites and articles. In Colombia every year changes are made in the tax regulations, since in very few occasions the money they have planned is collected and this happens, due to the lack of information from both natural and legal persons, since they do not pay taxes corresponding, they manage to escape from these, however, it is not only the citizens, because most of them do do their duty, it is also the state that is largely very corrupt and does not have long-term planning. With the new taxes that it adds or withdraws, therefore, there are changes in these norms, since they do not fulfill their purpose, which is to stimulate the growth of the country, equity and give support to all entrepreneurs to promote employment. To conclude, we show that the last government of Iván Duque has complied with some of the changes proposed in the reforms, on the other hand, it has made reforms per year, which does not benefit the country, because they do not adapt to the first reform, when you are already entering the second. However, this government has gone through the economic crisis that Covid-19 has brought with it and has plans for its third reform, since the money collected was invested for the well-being of citizens and their livelihood while the pandemic was happening. A structural tax reform is needed for the nation, but one that has a long-term vision.

Keywords: Tax reforms, effects, financing law, Colombia. 


\section{Introducción}

En Colombia se han creado muchas reformas tributarias y muy pocas han durado en el país, ya que el fin de estas es "principalmente estimular el desarrollo del mercado de capitales, reactivando el ahorro y la inversión de la economía. También, se esperaba subsanar la disminución de los ingresos del gobierno, ante la reducción de las tasas arancelarias." (González \& Calderón, 2002), es decir, en ellas nos estamos encaminando hacia los cambios estructurales de la tributaria, en donde las referencias que tenemos son los impuestos como el IVA, el impuesto sobre la renta, el impuesto sobre bienes inmuebles, entre otros; de ellos se recoge el dinero para el subsistir del país, sin embargo, la cantidad de cambios que se hacen en estos impuestos se debe a que nunca alcanza el dinero para el desarrollo e inversión en la nación.

El propósito de esta investigación es analizar los cambios que se han generado en los impuestos de nuestro país, por las reformas tributarias hechas en los últimos 3 años y por supuesto, teniendo en cuenta un poco de historia de los años noventa donde la economía del país dio grandes cambios en ello, donde el objetivo primordial era la modernización y vincularse con los mercados internacionales para un mayor recaudo financiero, en ese año se enfocaron en lo que fue mayor oportunidad laboral, darle inicios a la comercialización y también grandes cambios fiscales. De igual manera, se tiene en cuenta los años siguientes y lo que fue importante en estos.

Con el gobierno de Iván Duque se crearon dos leyes de financiamiento ley 1943 de 2018 y la ley 2010 del 2019 las cuales profundizaremos haciendo unas pequeñas comparaciones con las anteriores y que tanto han afectado al país. Actualmente con la pandemia, se ha comprobado que se ha cumplido la devolución del IVA, los tres días sin IVA al año y entre otras, pero, se analizara que efectos o consecuencias trae esto para todos los colombianos y los probables cambios en el futuro.

Del mismo modo, se supone que las reformas deben generar para un país tres pilares, como "la eficiencia en el recaudo, minimizando los costos, eficiencia económica debe evitar gravar en exceso la inversión y el ahorro y distorsionar en forma inconveniente la asignación de los factores de producción; y la equidad, que son dos contribuyentes en circunstancias similares deben pagar impuestos similares y quienes tienen mayor ingreso o riqueza deben contribuir en mayor proporción" (Perry, 2019). Se tendrá en cuenta si las nuevas reformas alcanzan estos pilares, puesto que varios investigadores recomiendan dejar de hacer tantos cambios y crear una norma tributaria fija para así conseguir en algún momento la equidad.

\section{Desarrollo}

\section{¿Por qué se ocasionan las reformas tributarias?}

En Colombia existía un promedio de cada 2 o 3 años se realizaban cambios en las normas tributarias, actualmente se presenta cada año una variación en estas, lo que genera todo lo contrario que se busca con ellas que es el bienestar para todos los ciudadanos y una adecuada recolección de impuestos para el subsistir de la nación. Las reformas han promulgado más desempleo, más pobreza, desigualdad total (Ahumada, 2016) y por eso, no se encuentra un punto de equilibrio para las reformas que se implementan, pero se visualiza que no hay un avance en el bienestar para todos.

El desempleo es una de las principales causas del país, se ve que no hay un buen desarrollo y Colombia es uno de ellos, porque si no hay trabajo no hay sustento y aumenta la 


\section{6}

criminalidad en las ciudades del país como en Cúcuta que es una de la ciudades con un alto índice de desempleo; (Acevedo Vanegas \& Montoya Montoya, 2019) todo lo negativo que dejan algunas reformas tributarias es por la mala planificación, en donde no hay un estudio detallado en que se verían los colombianos afectados si aumentan unos impuestos, si no que velan por el bien común del estado en donde buscan recaudos para ello.

Una de las causales primordiales para la alteración de estas normas es la renta petrolera, el petróleo es un sumamente importante en los ingresos de nuestro país, ya que, si este está en un gran incremento o auge, no posee el país los recursos suficientes para invertir y cumplir con los que ha estipulado en su gobierno. Otros impuestos que se recolectan es la renta y lucro ocasional, sobre la renta de la equidad, valor agregado, al consumo, sobre transacciones financieras, sobre la industria y comercio y predial. (Anselmo, s.f.).

Actualmente, se considera que se realizan varias reformas tributarias por la falta de planificación de estas, ya que esto impide que la primera reforma que se hace funcione a largo plazo. Puesto que, la manera que encuentra más factible el gobierno para la recolección de dinero, es el aumento de los impuestos. También se consideran estas complejas, porque muy pocos ciudadanos comprenden o conocen cuales son los impuestos y como se deben pagar; ineficientes, pues el estado no recoge lo necesario o imprescindible; inequitativo, debido a que la mayoría de la carga cae sobre las personas naturales y no sobre las grandes industrias (Hernandez Torres \& Martinez Quenza, 2020).

Como mencionamos anteriormente también se realizan estas reformas por la dependencia que tiene la obtención de "recursos provenientes de la venta de hidrocarburos, materias primas y regalías (Hernandez Torres \& Martinez
Quenza, 2020)". Una de las causas principales de los cambios constantes de estas normas es la evasión que realizan los ciudadanos para el pago de los respectivos impuestos, sin pago de estos, el estado no obtiene los recursos que necesita para cumplir con lo que estipula en el gobierno que este rigiendo.

Estas son algunas causas por las que se hacen cambios tributarios en el país, ya que también se puede mencionar la corrupción que tienen los gobernantes, las deudas externas y el descontrol del PIB del país. Como mencionamos anteriormente también hablamos de incumplimiento legal, debido a que no pagan lo correspondiente o siempre hay ciertos descuentos por parte del estado a los contribuyentes.

\section{Historia de las reformas tributarias}

Cuando se hace referencia a tributación se enfocan sobre los impuestos que nosotros como personas naturales, organizaciones o negocios deben pagar al estado nacional por conceptos de poseer alguna propiedad, adquirir un producto por medio de una compra o bien sea un servicio, pero estos impuestos no tienen que cancelar el mismo monto de dinero la persona o la empresa si no el monto a pagar lo define según sus activos que tengan o su patrimonio que tenga cada uno, ya si desean cambiar los valores de estos impuestos solo dependerá del estado del país como este manejando sus políticas y como se está viendo a nivel monetario y este cambio se hará mediante una reforma tributaria.

Lo que busca una reforma tributaria es un cambio ya sea aumentar o disminuir los montos monetarios que ir a recoger el estado el cual son pagados por medio de los impuestos que tiene cada persona o empresa mencionadas anteriormente. 
Un ejemplo de nuestro país que es un estado donde la corrupción es evidente, donde varias empresas evaden los impuestos, no llevan un control, un registro de las finanzas claras; donde la población no tiene un bienestar, la pobreza cada día es más clara por la falta de empleo y la economía de nuestro país no es el mejor debido a las malas inversiones que hacen nuestros mandatarios es donde es evidente que las reformas tributarias aumentaran los impuestos para equilibrar todo lo mencionado anteriormente.

En donde nuestro Estado Colombiano todo fuera diferente las reformas tributarias disminuían los impuestos que pagamos los colombianos, no haría la necesidad de que el estado recibiera todo aquello que le entra en conceptos monetarios por lo mismo.

En nuestro país ya han realizado varias reformas tributarias en donde no se motivaron a garantizarle trabajo a la ciudadanía, en donde la pobreza es algo preocupante en nuestro país y no le prestan mayor atención; en donde la desigualdad abarca todo donde los que tienen en abundancia quieren más y quieren ver a los pobres más pobres; todo esto cambiaria si las siguientes reformas se evidencian o se focalizan en hacer un estado progresivo en donde las oportunidades de conseguir un trabajo digno tanto para los jóvenes como para los adultos sea lo primordial y brindar mayor oportunidades.

Las nuevas reformas tributarias comprenden hacer el estado se basan en cinco artículos que son:Impuestos a las personas naturales, normas anti evasión y anti elusión, IVA, impuestos a las sociedades y ganancias ocasionales.

Se proponen reformas estatales que supuestamente buscan una mayor eficacia, pero se dejan de lado los procesos históricos y políticos inherentes al proceso, Carlos Villas pone de flagrante de cómo se forma la anticipación a los elementos de administración, en mano que se pretende disminuir la política a la delegación. Se da a entender en que una buena administración pública la debe ejercer un estado con objetivos claros y con una estructura bien planteada y en que las reformas económicas emprendidas sean soluciones para el país y seguir con el desarrollo (Ahumada, 2016), este modelo que establece Villas es a base de que se centre en el desarrollo económico como social en donde ahí iríamos a luchar contra la pobreza y un mejor bienestar para los más vulnerables y también se fijaría en dar más oportunidades laborales para que las personas puedan tener su sustento, porque ese es un problema que también está afectando al país como lo es el desempleo, la ciudad de Cúcuta es una de principales ciudades del país con el índice más alto de desempleo es por ello que hay mucha pobreza en esta ciudad y con el tema de la frontera con Venezuela que hay demasiado extranjero en nuestra ciudad les quita el poco trabajo que hay para los cucuteños es una problemática que vive en la actualidad hoy la ciudad y el país.

(Acosta Herrera, Mejía Larrea, Montoya Gallo, \& López Uribe, 2012) Nos imparte que las reformas tributarias son una remesa de puros gravámenes que los componen una secuencia de partes de caracteres jurídicos como también monetarios, el objetivo primordial que tienen o lo que todos pensamos es que las reformas son estudiadas, planificadas para acoger recursos para mantenimiento y desarrollo público del país y también a combatir las acciones sociales de la ciudadanía en donde el espacio público se ve afectado cuando se hacen marchas, manifestaciones y a veces se salen de control a rematar con lo que es el patrimonio del país, pero los cuatro principales objetivos que tienen las reformas son: la Neutralidad es decir que los ingresos que se van a utilizar para una obra en particular no afecte a la ciudadanía y que ese dinero no se pierda, como segundo tenemos la Eficiencia es decir que los objetivos 


\section{8}

propuestos a un determinado periodo de tiempo se cumplan o en el menor tiempo posible pero que se terminen cumpliendo, tercero la Equidad toda la población tiene que recibir de acuerdo a sus necesidades que los aquejan y como cuarto tenemos la Incidencia es donde se evidencia los casos que evaden dichos impuestos y es donde toca reducir ese inconveniente. En la ley 633 de la reforma del 2000 el IVA se lo gravaron a lo que fue el transporte aéreo a los vuelos internacionales, transporte público, a los cigarrillos y por ultimo a los tabacos con una tasa del $16 \%$ en donde el impacto del PIB fue del 1.8\%.

(Sanchez \& Espinosa, 2005) El autor citado nos habla que un determinado periodo de tiempo que fue desde 1980 hasta el 2003 se realizó en total 8 reformas en donde el objetivo enfocado que tenían era tener aumentos en los ingresos tributarios para lograr equilibrar la balanza los gastos monetarios por conceptos públicos en lapsos de tiempos cortos, al realizar una comparación de los datos del año 1980 al 2003 se vio muy notable que los ingresos se crecieron en mayor parte a la que se tenía esperanzado y es un estudio satisfactorio para el país que mediante por 8 reformas lograron para seguir sosteniendo nuestra economía. En la reforma del 2003 se enfocaron en tres puntos fundamentales que fueron suplir incentivos tributarios, se aumentaron los impuestos que para las personas naturales es algo complicado y como tercero tomaron medidas drásticas para controlar la evasión de lo mismo.

En la reforma del año 2006 se lleva a cabo la inexistencia de la tasa del $10 \%$ sobre el impuesto de a la renta en donde fue estipulada en la reforma del 2003 anteriormente mencionada tanto para las personas naturales como organizaciones en cual estarían en vigencia desde el 2004 hasta el 2006 y lo que hacen es aumentarla el triple la tasa en un $35 \%$ hasta un $38 \%$ sobre el impuesto de la renta en donde esta método fue utilizado por autor (Villarreal Cifuentes \& Peñuela Boorquez, 2006) en donde definía la tasa marginal como la diferencia del rendimiento capital antes de impuesto y el impuesto regido por el ahorrador y todo eso lo dividía entre el capital antes de impuestos , también el IVA paso del $10 \%$ a una tasa del $16 \%$ en algunos bienes y el impacto del PIB fue del $-0.4 \%$.

Nuestro país en los últimos 10 años (Dinero, 2015) se ha visto muy mal en el sistema monetario eso le da a lo consiguiente de que en las dos décadas que hemos transcurridos se hayan realizado doce reformas tributarias y esto comenzó desde los noventa en donde el mandatario de esa época era el doctor Cesar Gaviria y en años atrás el presidente Juan Manuel en donde la reforma que implanto en el año 2016 que fue donde Colombia también tuvo un crecimiento por medio de recaudos de la misma con el pro de que más profesionales, organizaciones entren en el mundo de declarar renta y es donde el pueblo no se siente bien viviendo en este país por el alza de los impuestos, cada vez que hacen una reforma se baja el número de declarantes esto quiere decir que las personas optan por trabajar en la informalidad ya que no tienen que rendirle cuentas al estado y lo que antes declaraban les queda a ellos.

Los autores (Tobar Martínez, Contreras Rodríguez, Bernal García, \& Cortes Devia, 2017) nos da una breve historia y es que los inicios o donde comenzaron hablar sobre tributación fue en la época de la edad media fue ahí donde se informaba lo esencial en nuestro Colombia ya transcurrido un lapso de tiempo llego al mandato el señor Francisco de Paula Santander en el año 1820 y el adopta la metodología inglesa y predetermina la contribución directa la cual no fue muy satisfactoria debido a que en esa época bélica no estaban en un momento armónico, más adelante después de transcurrir casi cien años es donde implantan 
el impuesto a la renta que se llamaba en esa época el impuesto a la conquista porque lo que trabajan los obreros esos dineros se recaudaban y con eso se les pagaba y su tasa empezó con un 50\% respecto al salario y luego fue mermando hasta lograr una tasa conveniente para todos y después como anteriormente mencionado llego el impuesto a la renta. (Clavijo, 2007) El autor mencionado históricamente nos habla de que nuestro país viene presentando un déficit a nivel fiscal debido al desorden del gasto público, el PIB está en un 3-6\% y el resto está en lo que son los pagos corrientes, es decir que nuestro país viene en mal en peor desde el 95 en donde lo único que hacen es endeudarse debido a una mal planeación e administración de los recaudos aunque en algunos años no se vio tanto el déficit pero seguían teniendo deudas en el cual el PIB registraba un 1.5\%, una realidad que si notamos es que el déficit fiscal q tenemos en nuestra administración, todo esto es evidente por medio de la inestabilidad de los pocos ingresos que obtiene el país y el mayor gasto que tenemos que es el gasto público y esto ya se viene presentando desde años atrás son los temas tributarios que más incomodan al gobierno (Riofrio Bonilla \& Sinisterra Duran, 2012) y no solo el autor mencionado está de acuerdo de que el desequilibrio de lo anteriormente es evidente y esto es duradero y probable dependiendo de cómo se esté llevando el registro al gasto público.

En general la reforma que marco un gran impacto fue la de la ley 78 del 35 (Mora Toscano, 2013) en esta reforma lo primordial fue que la base era la democracia en donde se viera en evidencia la progresividad tributaria en donde la medida era aumentar los impuestos directos y poder lograr cambiar el pensamiento de los contribuyentes como personas naturales como organizaciones, en esa contribución se iba a llevar unas pérdidas de los recursos económicos del país.
(González \& Calderón, 2002) Los logros tributarios tuvieron un notable alza durante los años noventa, las reformas tributarias, casi una al año durante la última década, se dio en una respuesta reactiva al crecimiento del gasto gubernativo, en donde se llega a enfrentar a la circunstancia de déficit monetario, ya que el gasto de la presidencia es esencial, y esto ha venido creciendo excesivamente, pero no se puede asegurar que ninguna de las reformas tributarias haya contribuido a dar soluciones estructurales al creciente compromiso del gobierno decisivo, es decir que en 1990 cuando se dio la apertura económica en Colombia los ingresos por concepto tributario se vieron reducidos de una manera descomunal ya que esto se vio reflejado en los que son llamados gravámenes arancelarios por lo consiguiente toco hacer nuevas reformas donde tocaba incrementar si o si los recursos.

\section{Reformas tributarias de los años 2018 a 2020}

En el año 2018 Colombia tiene como presidente a Iván Duque Márquez en este gobierno se han realizado dos reformas tributarias "pues en el primer año de su mandato (2018) creó la Ley de Financiamiento, y el año pasado la Ley de Crecimiento (2019) (Amaya, 2020)". En la ley 1943 de 2018 que es la primera que se realiza en esta se aprobó un régimen especial para las empresas que en cinco años realicen inversiones de más de un billón de pesos, tendrán beneficios especiales como: "Una tarifa reducida del impuesto de renta, eximirlos del impuesto al patrimonio y a los dividendos, no aplicarles renta presuntiva y permitirles la depreciación de sus activos fijos en solo dos años" (Barreto, 2019).

De igual manera, buscaba con las personas naturales recaudar 1,9 billones de pesos, puesto que aumento el impuesto sobre la renta para aquellos con ingresos superiores a 33 millones de pesos mensuales. En las personas 


\section{0}

jurídicas, deberán pagar un impuesto de utilidades de $7,5 \%$, pues esperan recaudar 368 millones de pesos. También se incluyó una sobretasa de renta del $4 \%$ para las entidades financieras y con ellas se espera recaudar hasta 2 billones de pesos en los 3 años (Silva Correa, 2019). Hubo servicios excluidos del impuesto a las ventas, como lo son la salud, es decir, servicios médicos, odontológicos, laboratorio y hospitalarios. Del mismo modo, los servicios a la educación de las instituciones públicas y privadas.

"Es importante resaltar cambios significativos como el impuesto sobre la renta para personas naturales; mayor control en la evasión fiscal; actualizaciones al impuesto de consumo para franquicias que se realicen en el país; cambios en la base gravable del IVA; se mantiene como un impuesto no constitutivo de renta los aportes voluntarios al sistema general de pensiones; creación del impuesto al patrimonio entre otros" (Ortega Gómez, 2018)

Resumiendo, los aspectos que encontramos en la ley de financiamiento son la extensión del IVA, incluyendo alimentos y excluyendo vivienda, educación, saludy servicios públicos, con un IVA del 19\%; devolución del recaudo por IVA a personas de menores ingresos; reducción de la tasa nominal a las utilidades de las empresas; reimposición del impuesto al patrimonio a las personas naturales de patrimonios elevados y mantenimiento del gravamen a las transacciones financieras en las tasa de 4 por mil (Ferrari, 2018).

A finales del año 2018 se declara la ley 1943, como inexequible, por ello, hay nueva reforma y recibe el nombre de "Ley de crecimiento" esta incorpora nuevos cambios, "en materia de IVA, del impuesto nacional al consumo, en la tributación de renta de personas naturales, frente a los dividendos y participaciones, en el impuesto de normalización, en el impuesto sobre la renta, en la declaración de activos en el exterior, en el GMF y en algunos aspectos procedimentales." (Legis, 2019). Igualmente, esta sigue teniendo todo lo referente a lo exención del IVA a personas de escasos recursos y devolución de este.

Con la actual pandemia, se ha realizado la implementación de la devolución del IVA poco a poco, también se realizaron los tres días propuestos sin IVA, el estado hizo descuentos en impuesto como prediales y de vehículos para facilitar la recolección de este dinero. Sin embargo, para nadie es un secreto que el país actualmente se encuentra en una crisis financiera debido a las ayudas que ha brindado a la población, a que no ha habido tanto comercio o ventas, el desempleo es mucho mayor que en años anteriores y por esto se habla de una tercera reforma en el actual gobierno, que vendría para el año 2021.

\section{Conclusión}

El fin de la realización de estas reformas en nuestro país por lo general es buscar reformar el estatuto tributario, en la mayoría de los casos para conseguir más ingresos públicos que le permitan adelantar más programas sociales y obras de desarrollo (vías, colegios, hospitales, subsidios a los más pobres, como Familias en Acción) (Tiempo, 2019). Por otra parte, también se hacen modificaciones con otros objetivos: "como promover el empleo, para lo cual les bajan los costos a las empresas por los impuestos que estas deben aportar para que así puedan producir más y así necesitar más trabajadores. Es por esa razón que en cada gobierno hay mínimo una reforma tributaria" (Tiempo, 2019).

Sin embargo, no todos los ciudadanos colombianos tienen conocimiento de estas reformas y como se deben pagar los impuestos, por ello, lo más pertinente para 
Colombia es realizar una reforma tributaria estructural, que sea proyectada a largo plazo y en ella se especifique lo que las personas naturales y jurídicas tienen la obligación de pagar, puesto que siempre se busca el aumento de empleo con la disminución de impuestos en las organizaciones y sucede lo contrario. De igual manera, se debe trabajar muy duro en contra de la corrupción tanto en los gobernantes, como en las diferentes entidades que son las encargadas de dar los beneficios económicos, debido a que estos recursos no llegan completos.

También se debe incentivar a las personas a pagar los impuestos que les corresponde, ofrecer cursos o materias en universidades y colegios, sobre la importancia que tienen estos para el sustento del país, del mismo modo dar a conocer en qué se gastan todos los dineros recolectados, buscar la equidad y no la igualdad, mejorar el sistema del sisben y demás para aquellos que reciben estas supuestas ayudas por parte del gobierno. Sin embargo, hay que derrotar poco a poco la corrupción, pues sin esta el dinero por fin rendiría y no habría necesidad de tantos cambios tributarios en el país.

\section{Referencias}

Acevedo Vanegas, J., \& Montoya Montoya, S. (2019). Reformas tributarias y sector vivienda: un análisis al caso colombiano. Obtenido de Tecnológico de Antioquia Institución Universitaria: https://dspace.tdea.edu.co/ bitstream/handle/tda/587/Reformas $\% 20$ tributarias $\% 20 \mathrm{y} \% 20$ sector $\% 20$ vivienda. pdf? sequence $=1$ \&isAllowed $=\mathrm{y}$

Acosta Herrera, L. J., Mejía Larrea, C. A., Montoya Gallo, J. E., \& López Uribe. (2012). ¿Han sido eficientes y exitosas las reformas tributarias del 1990-2009? Obtenido de Universidad de Antioquia: https://www.redalyc.org/ pdf/861/86129686009.pdf
Ahumada, C. (Junio de 2016). La propuesta de la Comisión para la reforma Tributaria. Obtenido de Friedrich-Ebert-Stiftung.: https://library.fes. de/pdf-files/bueros/kolumbien/12654.pdf

Amaya, J. (19 de Agosto de 2020). Las bases de la tercera reforma tributaria de Iván Duque se conocerán en febrero de 2021. Obtenido de La Republica: https://www.larepublica.co/ economia/para-que-se-creo-la-comision-deexpertos-en-beneficios-tributarios-reformatributaria-en-2021-3046756

Anselmo. (s.f.). ¿Cuáles son los impuestos en Colombia? Obtenido de Movistar: https:// destinonegocio.com/co/emprendimiento-co/ cuales-son-los-impuestos-en-colombia/

Barreto, L. (14 de Enero de 2019). Impuestos: ipor qué tantas reformas... y tantos subterfugios? Obtenido de La Razon pública: https:// razonpublica.com/impuestos-por-que-tantasreformas-y-tantos-subterfugios/

Clavijo, S. (Enero de 2007). EVOLUCION DE LA TRIBUTACION EN COLOMBIA Y SUS DESAFIOS (1990 - 2006). Obtenido de https:// www.cepal.org/ilpes/noticias/paginas/2/27472/ clavijo0107.pdf

Dinero. (2015). REVISTA ECONOMICA DINERO.

Ferrari, C. (5 de Noviembre de 2018). La reforma tributaria del presidente Duque. Obtenido de La razón pública: https://razonpublica.com/lareforma-tributaria-del-presidente-duque/

González, F. A., \& Calderón, V. (2002). Las reformas tributarias en Colombia durante el siglo XX (II). Obtenido de Boletines de divulgación económica, 9.: https://colaboracion. dnp.gov.co/CDT/Estudios\%20Econmicos/ Las\%20reformas\%20tributarias\%20en\%20 Colombia\%20durante\%20e1\%20sig10\%20 XX\%20(II).pdf 


\section{2}

Hernandez Torres, S., \& Martinez Quenza, O. S. (Agosto de 2020). ¿Por qué en Colombia se hacen tantas reformas tributarias? Obtenido de Universidad Cooperativa de Colombia: https://repository.ucc.edu.co/ bitstream/20.500.12494/20098/1/2020_ Colombia_reformas_tributarias.pdf

Legis.(30 de Diciembre de 2019). Ley de crecimiento económico “Reforma Tributaria2019”. Obtenido de Legis: http://www.comunidadcontable. com/BancoConocimiento/Otros/reformatributaria-2020.asp?Miga $=\&$ CodSeccion $=$

Mora Toscano, Ó. (Julio de 2013). La reforma tributaria de 1935 y el fortalecimiento de la tributación directa en Colombia. Obtenido de Universidad Pedagógica y Tecnológica de Colombia: https://www.redalyc.org/ pdf/4795/479549579003.pdf

Ortega Gómez, E. (2018). Implementación de la ley de financiamiento comercial. Obtenido de FITEC: http://www.fitecvirtual.org/ojs-3.0.1/ index.php/clic/article/view/308/271

Perry, G. (2019). Hacia una reforma tributaria estructura. Obtenido de Fundación para la Educación Superior y el Desarrollo (Fedesarrollo): https:// www.repository.fedesarrollo.org.co/ bitstream/handle/11445/3976/Co_Eco_ Junio-Diciembre_2019_Perry_Hacia. pdf?sequence $=1 \&$ isAllowed $=\mathrm{y}$

Riofrio Bonilla, M., \& Sinisterra Duran, J. (2012). Historia de las reformas tributarias aprobadas en Colombia entre los años 2000 y 2009. Obtenido de Universidad del Valle: https:// bibliotecadigital.univalle.edu.co/bitstream/ handle/10893/10698/CB-0504266.f;jsessionid= 78DEE4E64FB699D7AFA8C6C23DE57F42?s equence $=1$

Sanchez, F., \& Espinosa, S. (Febrero de 2005).
IMPUESTOS Y REFORMAS TRIBUTARIAS 80-03. Obtenido de CEDE: https://imgcdn. larepublica.co/cms/2014/09/18235605/ I m puest os $\% 20$ y $\% 20$ reformas $\% 20$ tributarias\%20-Cede.pdf

Silva Correa, A. N. (5 de Diciembre de 2019). Analisis del impacto de la nueva ley de financiamiento ley 1934 de 2018. Obtenido de Universidad de Cundinamarca: http:// repositorio.ucundinamarca.edu.co/ bitstream/handle/20.500.12558/3192/ A N \% c $3 \% 80$ L I S I S \% 20 D E L \% 20 IMPACTO \%20DE\%20LA\%20NUEVA\%20 LEY\%20DE\%20FINA NCIAMIENTO. pdf?sequence $=1 \&$ isAllowed $=\mathrm{y}$

Tiempo. (19 de Diciembre de 2019). Todo lo que hay que conocer sobre una reforma tributaria. Obtenido de El Tiempo: https://www.eltiempo. com/economia/sectores/reforma-tributariaque-es-y-que-propone-el-gobierno-en-lade-2019-445110

Tobar Martínez, J., Contreras Rodríguez, T., Bernal García, Y., \& Cortes Devia, L. (2017). ANÁLISIS HISTÓRICO DE LA REFORMA TRIBUTARIA: BREVE ASOMO A SU IMPLEMENTACIÓN EN COLOMBIA. Obtenido de Universidad Cooperativa de Colombia: https://repository.ucc.edu.co/ bitstream/20.500.12494/4783/1/2017_analisis_ historico_reforma.pdf

Villarreal Cifuentes, R., \& Peñuela Boorquez, M. (2006). Efectos de la reforma tributaria de 2006 sobre la tributación efectiva de las rentas del capital. Obtenido de Universidad de Antioquia: https://revistas.udea.edu.co/index. php/coyuntura/article/view/2319/1891 